\title{
On Payoff Heterogeneity in Games with Strategic Complementarities ${ }^{1}$
}

\author{
Antonio Ciccone \\ Universitat Pompeu Fabra and CEPR \\ and \\ James Costain \\ Universitat Pompeu Fabra \\ February 2002 \\ Corresponding author: \\ James Costain \\ Department of Economics and Business \\ Universitat Pompeu Fabra \\ Ramon Trias Fargas 25-27 \\ 08005 Barcelona, Spain \\ james.costain@econ.upf.es
} ${ }^{1}$ We thank Berthold Herrendorf, Akos Valentinyi, and Stephen Morris for helpful
comments. All errors are our own. 


\begin{abstract}
Payoff heterogeneity weakens positive feedback in binary choice models in two ways. First, heterogeneity drives individuals to corners where they are unaffected by strategic complementarities. Second, aggregate behaviour is smoother than individual behaviour when individuals are heterogeneous. However, this smoothing does not necessarily eliminate positive feedback or guarantee a unique equilibrium. In games with an unbounded, continuous choice space, heterogeneity may either weaken or strengthen positive feedback, depending on a simple convexity/concavity condition. We conclude that positive feedback phenomena derived in representative agent models will often be robust to heterogeneity.
\end{abstract}

JEL classification: C72, E00.

Keywords: Heterogeneity, multiplicity, discrete choice, strategic complementarity, positive feedback. 


\section{$1 \quad$ Introduction}

Many of the "new" theories in economics analyze the role of positive feedback. This is true of "new" growth theories as well as "new" trade theories. Positive feedback has aroused interest in economics since it may lead to multiple equilibria and since it may amplify the effect of exogenous variables on equilibrium outcomes.

Theories involving positive feedback are often developed using models in which all individuals are assumed to have identical objectives (payoff functions). ${ }^{2}$ The use of such models is usually regarded as an innocuous simplification. However, two recent papers claim that the lack of payoff heterogeneity is actually crucial for amplification and multiple equilibria. Schmutzler (1998) and Herrendorf, Valentinyi, and Waldmann (2000) construct games with a choice of two actions in which payoff heterogeneity dampens the effect of changes in exogenous variables on equilibrium outcomes (S98) and makes multiple equilibria less likely (HVW00). These conclusions are driven by the fact that payoff heterogeneity diminishes the strength of positive feedback in their models.

Our objective is to better understand whether and how payoff heterogeneity might affect positive feedback, and thus amplification and multiplicity. We first show that the results of S98 and HVW00 are driven by the fact that they focus on binary choice games. Positive feedback is affected by payoff heterogeneity in such games for two reasons. First, extreme heterogeneity implies that the utility implications of the two possible choices differ so much that, in the limit, each

\footnotetext{
${ }^{2}$ We focus on uncorrelated heterogeneity of the payoff function in full information games. Modelling heterogeneity as correlated shocks across individuals gives rise to some additional effects, not discussed here, which are particularly relevant in the case of incomplete information. See Morris and Shin $(1998,2002)$ and Chamley (1999).
} 
individual will take one of the two actions regardless of the choices of others. That is, extreme heterogeneity implies that each player is unaffected by the strategic complementarities which, as is well understood, are necessary for positive feedback and multiple equilibria (Cooper and John (1988)). Hence, extreme payoff heterogeneity always eliminates positive feedback and multiple equilibria in binary choice games. The second reason why payoff heterogeneity affects positive feedback in binary choice games is somewhat subtler. With two possible actions, each agent's strategy (as a function of others' behaviour) is characterized by threshold points at which the optimal choice jumps from one action to the other. Strong feedback results if many agents' behavior jumps in the same direction in response to the same aggregate change. However, this is improbable when individuals are heterogeneous, since heterogeneity is likely to imply different threshold points. Thus we show that there is a sense in which payoff heterogeneity smoothes aggregate behaviour in binary choice games. But we also demonstrate that this smoothing effect is not sufficient to eliminate positive feedback or to guarantee uniqueness.

Extreme payoff heterogeneity also eliminates amplification and multiplicity in continuous action games with a bounded choice set because it implies that, in the limit, all individuals are always on the boundary of their choice set independently of the behaviour of others. Hence, again, extreme heterogeneity means that individuals are unaffected by strategic complementarities. However, these claims about extreme heterogeneity are all limiting arguments which require imposing unchanging bounds on the choice space without imposing any bounds on the allowable degree of heterogeneity. Moreover, there is no sense in which payoff heterogeneity smoothes aggregate behaviour in continuous action games. We show that with a continuous choice set, positive feedback may either be amplified or weakened by heterogeneity, depending on a simple convexity/concavity condition. 


\section{A Game with Strategic Complementarities and Heterogeneity}

We ask how payoff heterogeneity affects feedback in a framework based on the simultaneous-move game of Cooper and John (1988), henceforth CJ88. This is the simple, transparent context in which the concept of strategic complementarities was first defined. While S98 and HVW00 work with dynamic models, in each paper the relevant properties of the dynamics turn out to hinge on the properties of an underlying static game equivalent to the one analyzed here, so little is lost by working with the static game itself. There is a continuum of individuals of measure one. An individual's payoff depends on her own action $x \in X$ and on the actions of others; for simplicity, we focus on the case where only the mean action $\bar{x}$ of others matters. Payoffs also depend on an individual characteristic $z$. The distribution of $z$ among individuals is given by $F(z)$, and the density, where it exists, is called $f(z)$. We consider equilibria of the game in which individuals simultaneously choose $x$ to maximize the payoff $V(x, \bar{x}, z)$.

We will make the following assumptions on the utility function:

$$
V_{x x}<0, V_{x z}<-b<0, \text { and } V_{x \bar{x}}>0
$$

We assume that $V$ and these derivatives are bounded and continuous. The derivative $V_{x z}$ will govern how strongly marginal utility is affected by the individual characteristic $z$. We bound $V_{x z}$ away from zero to ensure that differences in $z$ can have an impact on behaviour; otherwise heterogeneity in $z$ would be of no interest. ${ }^{3}$ The most important assumption here is that $V_{x \bar{x}}>0$ : a rise in the average choice $\bar{x}$ increases every individual's marginal utility of $x$. This is the assumption that CJ88 call strategic complementarities. Strategic complementarities are crucial for the way that average choices feed back upon individual choices, as we now show. 
Suppose all players believe that the average choice will be $\bar{x}$. The individual best response $y$ is given by

$$
y=g(\bar{x}, z)=\underset{x \in X}{\arg \max } V(x, \bar{x}, z)
$$

The average choice $\bar{y}$ which would be made in response to a conjectured average choice $\bar{x}$, which we will call the aggregate best response function, is then

$$
\bar{y}=G(\bar{x}) \equiv \int_{-\infty}^{\infty} g(\bar{x}, z) d F(z)
$$

The points where the aggregate best response function crosses the $45^{\circ}$ line are the equilibria of the game.

The slope of the aggregate best response function shows how individuals' behaviour, on average, responds to changes in average behaviour; hence it is natural to define this slope as our measure of feedback. What interests us is not only the feedback at any given point, but also the width of the interval over which a given average or minimum feedback applies. Greater feedback in an interval around a stable equilibrium implies that the equilibrium adjusts more in response to an infinitesimal exogenous shock (a larger "multiplier", in the sense of CJ88); and if the interval is wide, then there is a large multiplier not only in response to infinitesimal shocks, but also to "large" shocks, as illustrated in Figure 1a. Sufficiently strong positive feedback (at least equal to one) is also needed for multiplicity; and again the width of the interval of strong feedback matters, for it determines whether or not the two most widely separated equilibria differ significantly. If there exist two equilibria separated by distance $r$, then the average feedback over the interval between the equilibria must be one; also, if the average feedback on an interval of width $r$ which contains an equilibrium is $\phi>1$, then there

\footnotetext{
${ }^{3}$ Note that we focus throughout on heterogeneity that affects marginal payoffs and is therefore relevant for choice. Heterogeneity that affects only the level of payoffs has no fundamental effects.
} 
exist multiple equilibria separated by at least $\phi r$; see Figure $1 \mathrm{~b}$. Thus by asking how heterogeneity affects feedback we address simultaneously the size of the "multiplier", as in S98, and the likelihood and economic significance of multiplicity, as in HVW00.

The main conclusion of CJ88 is that strategic complementarities are necessary for positive feedback. They state their argument in the context of an interior solution of a continuous choice model. In this case, the individual best response $g(\bar{x}, z)$ is determined by the first-order condition

$$
V_{x}(x, \bar{x}, z)=0
$$

Assuming concavity of $V$ and strategic complementarities, (4) implies that a rise in the average choice $\bar{x}$ will increase the marginal utility of $x$, causing all individuals to increase their choice of $x: g_{\bar{x}}(\bar{x}, z)>0$. Since all individuals increase $x$, the aggregate best response function slopes upward, that is, there is positive feedback.

Note, though, that while strategic complementarities are necessary for positive feedback, they are not always sufficient. In this paper, we will want to consider both discrete choice problems and corner solutions to continuous choice problems. Hence (4) is not always the relevant condition to determine individual choice, and an infinitesimal change in average behaviour will not necessarily affect any given individual's behaviour. We will say that an individual is affected by strategic complementarities if there is some level of the aggregate choice $\bar{x} \in X$ at which the individual best response function $g(\bar{x}, z)$ is increasing in $\bar{x}$. Thus we immediately observe:

Proposition 1. If no individual is affected by strategic complementarities, then there is no positive feedback. 
That is, unless some individuals' best response functions are increasing on $X$, the aggregate best response function cannot be increasing on $X$. This simple fact will be crucial when we study the impact of heterogeneity.

\subsection{Effects of heterogeneity under binary choice}

We first consider the effects of heterogeneity when individuals can choose between two actions only, "left" and "right"; hence we define the choice set as $X=\{0,1\}$. Calling the choices 0 and 1 is a useful normalization because it means that the average choice $\bar{x}$ is equal to the measure $m$ of individuals playing $1 .^{4}$

Now suppose everyone believes that a measure $m$ of players will choose 1 . Then it will be optimal for an individual with characteristic $z$ to play 1 if

$$
V(1, m, z)-V(0, m, z) \equiv U(m, z) \geq 0
$$

The strategic complementarity assumption, in the binary case, implies that $U_{m}(m, z)>0$; that is, playing 1 is more advantageous when more people play 1 . Our assumptions on $V$ also imply that $U$ is bounded, with continuous first derivatives, and that the relative payoff to playing 1 is strictly decreasing in $z: U_{z}(m, z)<-b<0$. As before, bounding $U_{z}$ away from zero ensures that heterogeneity matters for choice. Thus at any $m$ there is a unique threshold $Z$ such that everyone with characteristic $z<Z$ plays 1 , while those with $z>Z$ play 0 . Individuals whose characteristic is exactly equal to the threshold value are indifferent between 0 and 1 . The threshold value $Z$, as a function of $m$, is implicitly defined by the equation $U(m, Z(m))=0$.

In this binary context, the aggregate best response function describes the measure $n$ of individuals who find it optimal to play 1 if everybody believes that a measure $m$ of individuals will choose 1 . Combining the threshold function $Z(m)$

\footnotetext{
${ }^{4}$ More precisely, if all individuals face the same two choices, calling these 0 and 1 is a normalization. But it is not without loss of generality to assume that all individuals have the same pair of choices.
} 
with the distribution $F(z)$ of characteristics in the population, our previous definition (3) of the aggregate best response function simplifies to

$$
n=H(m) \equiv F(Z(m))
$$

The aggregate best response function $H(m)$ maps [0,1] into itself. At least one equilibrium exists (given our assumptions on $U$ ), either at a corner, or at point where $H$ crosses the $45^{\circ}$ line continuously, or at a point where $H$ jumps across the $45^{\circ}$ line, in which case there is a mass of individuals who are indifferent between 0 and 1 in equilibrium.

Since the aggregate best response function is a transformation of the cumulative distribution function $F$, it is clear that heterogeneity may play an important role in determining feedback. Exactly how, though, do we define heterogeneity? There is no universally accepted definition, so we pick our definition for analytical convenience. ${ }^{5}$ We regard a population as heterogeneous if the density function is fairly flat, while a spike in the density, or a point mass, implies homogeneity. Hence a possible measure of homogeneity is the total absolute variation $A$ of the density function:

$$
A \equiv \int_{-\infty}^{\infty}\left|f^{\prime}(z)\right| d z
$$

Here we defined homogeneity over the whole real line, but it can also be usefully defined over a smaller interval, by changing the limits of integration. Notice that under our definition, $A$ is infinite if there is any point mass (a group of exactly identical people) in the distribution, and if we define $A$ over the whole real line, $A \geq 2 \max f(z)$. Thus also, increasing heterogeneity without bounds means driving

${ }^{5}$ Our definition of homogeneity is a special case of the definition proposed in Kneip (1999). By defining homogeneity, of course, we implicitly define its opposite, heterogeneity. 
the density function to zero everywhere. That is, a sequence of distributions $F_{i}$ for which $A_{i}$ approaches zero implies densities $f_{i}$ which approach zero pointwise.

We now see that the feedback at a given point $m$ can be decomposed into two factors, one relating to strategic complementarity, and the other to heterogeneity. Taking the derivative of the aggregate best response function, we have:

$$
\frac{\partial n}{\partial m}=H^{\prime}(m)=f(Z(m)) Z^{\prime}(m)
$$

The factor $Z^{\prime}(m)=-U_{m} / U_{z}$ is positive if and only if there are strategic complementarities, $U_{m}>0$. We can also say that the strategic complementarities are stronger when $Z^{\prime}(m)$ is larger, since this means a given change in the number choosing 1 has a bigger impact on the benefit of playing 1 . The factor $f(Z(m))$, the density of individuals at the threshold, captures purely "compositional" effects-it shows that there is stronger feedback at a point $m$ where many individuals choose to change their behaviour.

This decomposition helps clarify the two ways in which heterogeneity weakens feedback in binary models. Recall that increasing heterogeneity without limit (that is, lowering $A$ to zero) requires spreading out the density forever so that $f(z)$ approaches zero at each $z$. Therefore the number of people in the interval $[Z(0), Z(1)]$ must approach zero. Note that everyone outside the range $[Z(0), Z(1)]$ is unaffected by strategic complementarities, since someone with $z \leq Z(0)$ chooses 0 regardless of others' actions, and likewise, choosing 1 is a dominant strategy for anyone with $z \geq Z(1)$. Thus as we spread out the density function, in the limit nobody is affected by strategic complementarities, and from (8), feedback is zero at all possible $m$.

Proposition 2. Consider a binary choice model satisfying (1), characterized by some threshold function $Z(m)$. As we increase heterogeneity without 
limit, the number of individuals affected by strategic complementarities goes to zero.

Proof. Consider $U(0, z)$ for arbitrary $z$; this is finite by assumption. Since $U_{z}<0$ is bounded away from zero, there exists a finite $Z(0)$ satisfying $U(0, Z(0))=0$. Similarly, $Z(1)$ is finite. Hence since $Z(1)-Z(0)$ is finite, and since the density goes to zero everywhere as $A$ approaches zero, the mass in $[Z(0), Z(1)]$ goes to zero.

\section{QED}

This proposition implies that the slope of the aggregate best response function actually goes to zero at all points $m \in(0,1)$, so that not only is equilibrium unique, but also the multiplier effect of all shocks (within some range) is zero. As a corollary, continuity implies that for any $Z(m)$, there is some level of homogeneity $A^{*}$ such that $A<A^{*}$ implies a unique equilibrium, as shown in HVW00. ${ }^{6}$

This result is likely to be relevant for some contexts, but its generality is questionable since it arises only as we approach the limit. A great deal of dispersion of $z$ may be needed to eliminate multiplicity if $Z(m)$ is steep. Thus it is interesting to ask whether there is any other mechanism by which lesser degrees of heterogeneity also diminish feedback. The answer is yes, as we see by reconsidering equation (8). One way to generate strong positive feedback in a neighborhood of $m$ is to have a strong underlying strategic complementarity (large $Z^{\prime}(m)$ ). However, strong positive feedback can also result from a weak strategic complementarity, coupled with a dense concentration of individuals who would change their behaviour

\footnotetext{
${ }^{6}$ Morris and Shin (2002) discuss two types of sufficient conditions for uniqueness in imperfect information games: a large amount of independent heterogeneity, or a small amount of highly correlated heterogeneity. They point out that our propositions 2 and 5 are analogous to their "large heterogeneity" result, but have no bearing on their "small correlated heterogeneity" result.
} 
near $m$. In fact, if all the mass lies at some characteristic $z \in(Z(0), Z(1))$, then there are necessarily three equilibria. But this second source of positive feedback requires a spike in the density function around $m$-that is, it requires a fairly homogeneous distribution. More formally, if the average feedback on an interval of width $r$ is $\phi$, then the degree of homogeneity must be at least $A(\phi, r)$, which is an increasing function of $\phi$ and $r$.

As we prove this result, we hold fixed the number of individuals affected by strategic complementarities, $\sigma=F(Z(1))-F(Z(0))$, to emphasize that we do not eliminate the basic necessary condition for positive feedback. Therefore we compute homogeneity by integrating over the interval $[Z(0), Z(1)]$ only; the minimum homogeneity $A=0$ is now given by a uniform density on this interval. Now consider intervals of width $r$. Notice that average feedback over such an interval cannot exceed $\phi^{r}=\sigma / r$, which is the feedback obtained if all the individuals affected by strategic complementarities are in the interval. Also, there is a lower level of feedback achieved on some interval of width $r$ when mass $\sigma$ is uniformly distributed on $[Z(0), Z(1)]$; we will call it

$$
\phi_{r}=\max _{m \in[0,1-r]}\left(\frac{\sigma}{r}\right) \frac{Z(m+r)-Z(m)}{Z(1)-Z(0)}
$$

With these definitions, we have

Proposition 3. Consider a binary choice model satisfying (1), characterized by some threshold function $Z(m)$; fix the fraction of individuals $\sigma$ affected by strategic complementarities. (a) There is a minimum amount of homogeneity $A(\phi, r)$ required to construct average feedback $\phi$ on an interval of width $r . A(\phi, r)$ is strictly increasing in $r$ on $[0,1]$ and in $\phi$ on $\left[\phi_{r}, \phi^{r}\right]$. (b) If there exist two equilibria separated by distance $r$, then homogeneity must be at least $B(r)$, which is a strictly increasing function of $r$. 
Proof. Fix the mass of individuals $\sigma$ in $[Z(0), Z(1)]$. Consider an interval $I \equiv\left[Z\left(m_{0}\right), Z\left(m_{0}+r\right)\right]$ and an interval $J \equiv\left[Z\left(m_{0}-\delta\right), Z\left(m_{0}+r+\varepsilon\right)\right]$ such that $I \subseteq J \subseteq[Z(0), Z(1)]$. Consider the density $f$ which distributes mass $\sigma$ uniformly on $J$, and is zero elsewhere. Since $Z$ is continuous in $m$, both the homogeneity and the feedback associated with $f$ are continuous functions of $m_{0}, \delta$, and $\varepsilon$, and both are decreasing in $\delta$ and $\varepsilon$. Thus we can construct densities of this shape which attain every feedback between $\phi_{r}$ and $\phi^{r}$; note that no density can attain feedback greater than $\phi^{r}$ on an interval of width $r$. Hence there exists a minimum homogeneity $A(\phi, r)$ required to construct feedback $\phi \in\left[\phi_{r}, \phi^{r}\right]$ on an interval of width $r$. The envelope theorem implies that $A(\phi, r)$ is strictly increasing in $\phi$ and $r$. Proposition $3 \mathrm{~b}$ follows as a corollary, setting $\phi=1$. QED

This proposition states that if we restrict ourselves to more heterogeneous distributions, then a given level of feedback is only possible over smaller intervals, and less feedback is possible on intervals of a given width. This means that exogenous shocks are less likely to have a big effect, and that the most widely separated equilibria (if multiple) cannot be so far apart. This is simply because if many individuals have similar thresholds $Z(m)$, then aggregate behaviour exhibits a sharp jump at some $m$, just as individual behaviour does. But when $Z(m)$ differs across individuals, feedback is weakened, since aggregate behaviour is smoother than individual behaviour. This mechanism is similar to the one underlying Caballero's (1992) "fallacy of composition" result.

We should also note the limitations of this proposition. It refers to the minimum homogeneity required to construct (by choosing $F$ ) a certain amount of feedback over an interval of a given width. Hence it does not imply a monotonic relationship between heterogeneity and feedback for every conceivable change of the distribution. On the contrary, every change in $F$ (holding fixed $\sigma$ ) must lower 
feedback at some points and raise it at others; the effect on feedback around the equilibrium point(s) is ambiguous in general. Also, while increasing $f(Z(m))$ where $Z^{\prime}(m)$ is largest reinforces the underlying strategic complementarity by increasing the maximum feedback of the aggregate best response function, increasing $f(Z(m))$ where $Z^{\prime}(m)$ is small may increase homogeneity and yet decrease the maximum feedback, possibly eliminating multiplicity.

Moreover, while uniqueness is necessarily achieved when we drive the fraction of individuals affected by strategic complementarities to zero, the compositional effects of heterogeneity alone do not suffice for uniqueness. If we fix the number of individuals affected by strategic complementarities, then as we approach a uniform distribution on $[Z(0), Z(1)]$, we minimize homogeneity $A$ (as measured on the same interval). Nonetheless, since $Z^{\prime}(m)$ is arbitrary, feedback may still be strong, and there may still be multiplicity. The main necessary condition for multiplicity which we must impose on $Z^{\prime}(m)$, if $F$ is uniform on $[Z(0), Z(1)]$, is that $\max _{\mathrm{Z} \in(\mathrm{Z}(0), Z(1))} Z^{\prime}(m)>\sigma /(Z(1)-Z(0))$. Hence we have demonstrated:

Proposition 4. Consider a binary choice model satisfying (1), characterized by some threshold function $Z(m)$; fix the fraction of individuals $\sigma$ affected by strategic complementarities. (a) A more heterogeneous distribution may imply more or less equilibria than a less heterogeneous distribution. (b) Increasing heterogeneity up to a uniform distribution on $[Z(0), Z(1)]$ does not guarantee uniqueness.

Our results so far remind us, as we already know from CJ88, that the key ingredient for positive feedback is strategic complementarity. Multiple equilibria may be created by compositional effects under weak strategic complementarities, and this multiplicity will not be robust to heterogeneity. However, if the cause of multiplicity is strong strategic complementarity, then as long as we fix the number of 
individuals affected by these strategic complementarities, even a uniform distribution of $z$ (among those affected) does not eliminate multiplicity. Our uniqueness result arises only as we drive the fraction of individuals affected by strategic complementarities to zero.

\subsection{Effects of heterogeneity under continuous choice}

Next, consider how our results extend to the case of a continuum of possible actions. Proposition 2 eliminated feedback by driving individuals to corners where their behaviour was unaffected by others; hence we cannot expect any analogous proposition if the choice set $X$ is unbounded. But if we bound $X$, defining $X \in[0,1]$ (without loss of generality, subject to the same caveats as before), then sufficient heterogeneity will again eliminate the individuals who are affected by strategic complementarities. To see this, consider the threshold $Z_{0}(\bar{x})$ such that $V_{x}(0, \bar{x}, z)<0$ if $z>Z_{0}(\bar{x})$, and $V_{x}(0, \bar{x}, z)>0$ if $z<Z_{0}(\bar{x})$, and the analogous threshold $Z_{1}(\bar{x})$ such that $V_{x}(1, \bar{x}, z)<0$ if $z>Z_{1}(\bar{x})$, and $V_{x}(1, \bar{x}, z)>0$ if $z<Z_{1}(\bar{x})$. These are the values of $z$ at which the constraints $x \geq 0$ and $x \leq 1$ bind. Thus an individual is affected by strategic complementarities if and only if $\mathrm{z}$ is in the interval $\left[Z_{1}(0), Z_{0}(1)\right]$. Therefore:

Proposition 5. Consider a continuous, bounded choice model satisfying (1), characterized by threshold functions $Z_{0}(\bar{x})$ and $Z_{1}(\bar{x})$. As we increase heterogeneity without limit, the number of individuals affected by strategic complementarities goes to zero.

Proof. Our assumptions on $V$ imply that $Z_{0}(\bar{x})$ and $Z_{1}(\bar{x})$ are bounded and increasing functions, with $Z_{0}(1) \geq Z_{0}(\bar{x})>Z_{1}(\bar{x}) \geq Z_{1}(0)$, for $\bar{x} \in[0,1]$. Hence $Z_{0}(1)-Z_{1}(0)$ is finite. As homogeneity $A$ approaches zero, the density everywhere in $\left[Z_{1}(0), Z_{0}(1)\right]$ goes to zero. Note that individuals with $z$ outside this interval are 
unaffected by strategic complementarities, for they all prefer one particular corner regardless of $\bar{x}$. QED

We have shown that under our assumptions on $V$, there exist values of $z$ low enough so that individuals choose 1 regardless of the behaviour of others, and high enough so that individuals choose 0 regardless of the behaviour of others. Once again, spreading the distribution without limit eliminates those affected by strategic complementarities, making the feedback zero everywhere on $[0,1]$, and implying that there is some $A^{*}$ such that $A<A^{*}$ makes equilibrium unique. But as with Proposition 2, strong assumptions are required: we fix and bound the choice set, yet allow unbounded heterogeneity.

Is there any way in which weaker forms of heterogeneity also diminish feedback? In the continuous choice model, the answer is no. Individual behavior is not inherently discontinuous in this case, so we cannot construct a jump in aggregate behaviour through compositional effects, and there is no analogue to Proposition 3. Instead, calculating the slope of the aggregate best response function (2) for this case shows us a different way in which changes of the distribution affect feedback:

$$
\frac{\partial \bar{y}}{\partial \bar{x}}=G_{\bar{x}}(\bar{x}) \equiv \int_{-\infty}^{\infty} g_{\bar{x}}(\bar{x}, z) d F(z)
$$

This integral immediately implies (Rothschild and Stiglitz, (1971)):

Proposition 6. Consider a continuous choice model satisfying (1), characterized by the policy function $g(\bar{x}, z)$. The feedback at each $\bar{x}$ increases (decreases) in response to a mean-preserving spread of $F$ if $g_{\bar{x}}(\bar{x}, z)$ is convex (concave) in $z$ over the support of the spread. ${ }^{7}$

\footnotetext{
${ }^{7}$ By the "support of the spread", we mean the set of points $z$ at which the new distribution function differs from the old one.
} 
There are no economic reasons to restrict the convexity/concavity properties of the policy function slope $g_{\bar{x}}(\bar{x}, z)$ with respect to $z$, so the bottom line of Proposition 6 is that mean-preserving spreads affect feedback ambiguously. Note that although we do not view mean-preserving spreads as a good way of ordering heterogeneity in general $^{8}$, some decreases in homogeneity $A$ are mean-preserving spreads, so ambiguity with respect to mean-preserving spreads also implies ambiguity with respect to our homogeneity measure $A$. Hence Proposition 6 shows that for a continuous, unbounded choice space, an increase in heterogeneity may increase or decrease feedback at some or all points. The same conclusion applies when the choice space is continuous and bounded, if we hold fixed the fraction of individuals unaffected by strategic complementarities (those outside $\left[Z_{1}(0), Z_{0}(1)\right]$ ). For still more ambiguity, note that the equilibrium point(s) $x^{*}$ may move when the distribution changes, so that even if we can sign the effect on the feedback at all points, we are not sure what happens to feedback at the equilibrium point(s).

\section{Conclusions}

It has been proposed that we should be skeptical of economic arguments based on positive feedback, because, in some cases, feedback is weakened or eliminated by payoff heterogeneity. This paper has characterized the set of models in which this concern is justified. If we are willing to impose unbounded amounts of heterogeneity in payoffs, while fixing and bounding the choice set, then indeed, feedback is eventually eliminated. Also, we know that in the binary choice case, stronger feedback can be constructed in homogeneous models than in heterogeneous models. However, in continuous choice models, no such mechanism applies. Thus while there are some circumstances in which payoff heterogeneity weakens positive

\footnotetext{
${ }^{8}$ Compare a uniform distribution on $[0,1]$ to a pair of equal point masses at 0 and 1 . The latter is a mean-preserving spread of the former, but it is not necessarily convincing to say
} 
feedback, there is no reason to believe that representative agent models with positive feedback are vulnerable to heterogeneity in general.

\section{References}

CABALlERO, R. (1992), "A Fallacy of Composition", American Economic Review, 82, 1279-92.

CHAMLEY, C. (1999), “Coordinating Regime Switches", Quarterly Journal of Economics, 114, 867-905.

COOPER, R. and JOHN, A. (1988), "Coordinating Coordination Failures in Keynesian Models", Quarterly Journal of Economics, 103, 441-463.

HERRENDORF, B., VALENTINYI, A. and WALDMANN, R. (2000), "Ruling out Multiplicity and Indeterminacy: the Role of Heterogeneity", Review of Economic Studies, 67, 295-307.

HILDENBRAND, W. (1999), "Editorial”, Journal of Mathematical Economics, 31, $1-14$.

KNEIP, A. (1999), "Behavioural Heterogeneity and Structural Properties of Aggregate Demand", Journal of Mathematical Economics, 31, 49-79.

MORRIS, S. and SHIN, H.S. (1998), "Unique Equilibrium in a Model of SelfFulfilling Currency Attacks", American Economic Review, 88, 587-597.

MORRIS, S. and SHIN, H.S. (2002), "Heterogeneity and Uniqueness in Interaction Games", mimeo, Yale University.

ROTHSCHILD, M., and STIGLITZ, J. (1970), "Increasing Risk. I: A Definition”, Journal of Economic Theory, 3, 66-84.

SCHMUTZLER, A. (1998), "Changing Places- The Role of Heterogeneity and Externalities in Cumulative Processes," International Journal of Industrial Organization, 16, 445-461.

that it is more heterogeneous. 
Fig. 1a: response to non-infinitesimal parameter change

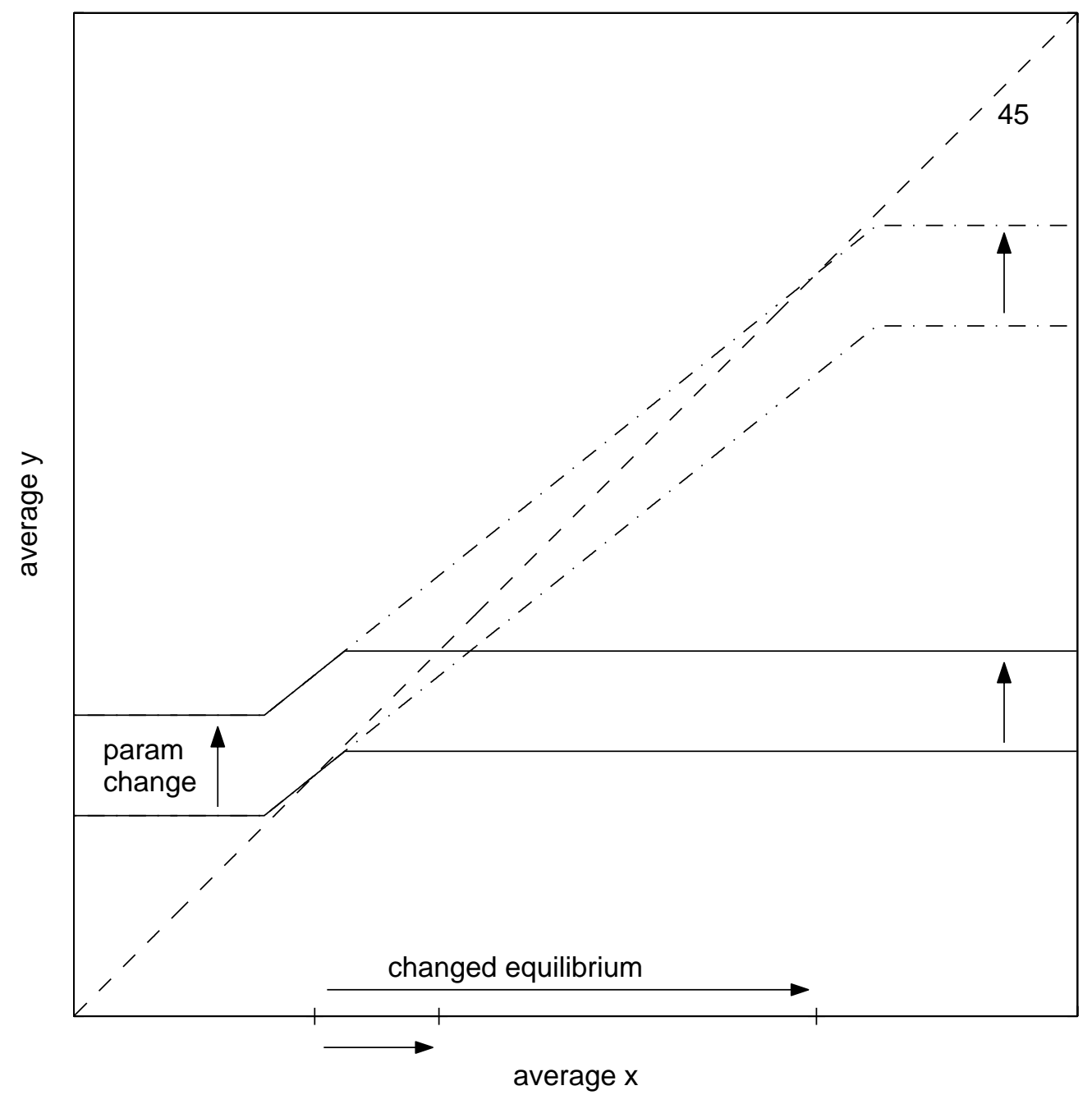


Fig. 1b: distance between equilibria

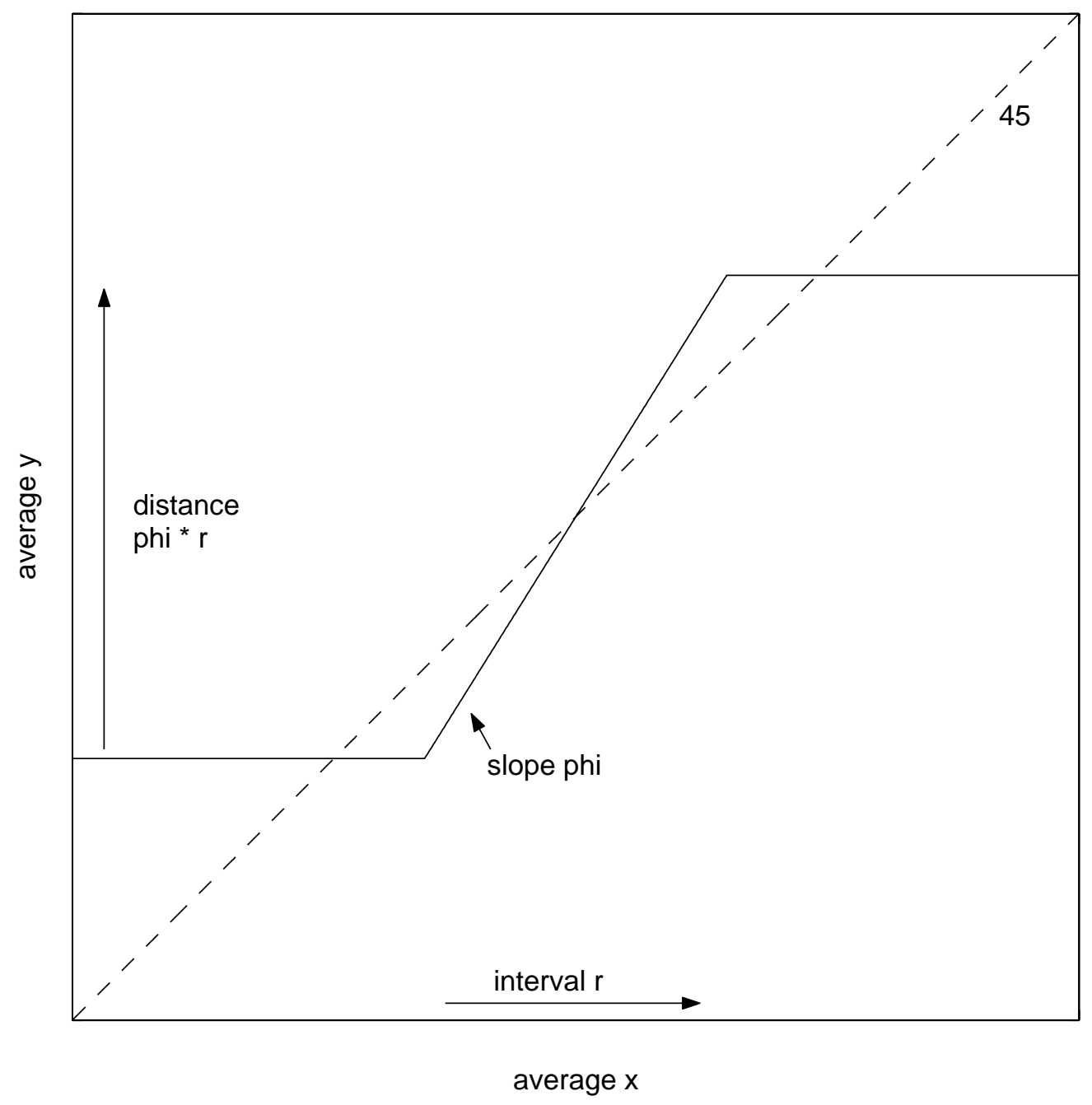

